\title{
LYAPUNOV CHARACTERISTIC EXPONENTS ARE NONNEGATIVE
}

\author{
FELIKS PRZYTYCKI
}

(Communicated by Clifford J. Earle, Jr.)

\begin{abstract}
We prove that, for an arbitrary rational map $f$ on the Riemann sphere and an arbitrary probability invariant measure on the Julia set, Lyapunov characteristic exponents are nonnegative a.e. In particular $\log \left|f^{\prime}\right|$ is integrable. An analogous theorem is proved for smooth maps of an interval with all critical points being nonflat.

This allows us to fill a gap in the proof of Denker and Urbański's theorem that there exists a probability conformal measure on the Julia set with exponent equal to the supremum of the Hausdorff dimensions of probability invariant measures with positive entropy.
\end{abstract}

\section{INTRODUCTION}

Our main aim is to prove the following

Theorem A. Let $f$ be a rational mapping of the Riemann sphere $\overline{\mathbb{C}}$ and $\mu$ an arbitrary probability $f$-invariant measure on the Julia set $J=J(f)$. Then for $\mu$-almost every point $x \in J$

$$
\chi(x)=\lim _{n \rightarrow \infty} \log \left|\left(f^{n}\right)^{\prime}(x)\right| \geq 0 .
$$

In particular, the function $\log \left|f^{\prime}\right|$ is $\mu$-integrable.

This easily yields

Corollary A. For $\mu$-almost every $x \in J, \lim \sup _{n \rightarrow \infty}\left|\left(f^{n}\right)^{\prime}(x)\right| \geq 1$.

The same methods (Koebe-like distortion lemma + the nonexistence of homtervals, which replaces Montels's Theorem) give

Theorem B. For every $f: I \rightarrow I$ a smooth map of the interval with a finite number of critical points all being nonflat and $\mu$ an arbitrary probability $f$-invariant measure on $\mathcal{J}^{\mathbb{R}}$, which denotes the complement of the domain of attraction to sinks and neutral points here, for $\mu$-almost every point $x \in J^{\mathbb{R}}$ the formula (1) holds.

One could ask whether, given $f, \chi(x)>0$ for every $\mu$ and $\mu$-a.e. $x$. Sometimes it is true; for example, it is obvious if $f$ is expanding on $J$ (i.e.,

Received by the editors December 16, 1991.

1991 Mathematics Subject Classification. Primary 58F23; Secondary 58F11, 58F08. 
if $\left|\left(f^{n}\right)^{\prime}\right|>1$ for some $\left.n\right)$. It is also true for subexpanding $f$, namely, such that the $\omega$-limit set $\omega(\operatorname{Crit} \cap J)$ is disjoint with Crit, where Crit $=\operatorname{Crit}(f)$ denotes the set of critical points for $f$, and there are no neutral rational points in $J$. This uses the recent Mañé's result that $f$ is expanding on $\omega($ Crit $\cap J)$ [M].

However, $\chi=0$ for $\mu$ supported by a "neutral set". Then we call $\mu$ a neutral measure. This happens, for example, for neutral points in $J(f)$ with Dirac measures on them, for harmonic measure on the boundary of a Siegel disc, or even in the case $J(f)=\overline{\mathbb{C}}$ for the invariant probability measure on the unit circle for some maps of the form

$$
z \rightarrow \lambda z \frac{z-a}{1-\bar{a} x}\left(\frac{z-\gamma a}{1-\gamma \bar{a} z}\right)^{-1} \quad \text { for }|\lambda|=|\gamma|=1, \quad \gamma \approx 1 ;
$$

see [H, GPS].

Corollary A is useful already in a weaker form, namely, that there exists at least one $x$ in every closed forward invariant subset of $J$ such that lim $\sup _{n \rightarrow \infty}\left|\left(f^{n}\right)^{\prime}(x)\right| \geq 1$. This fact allows one to prove that the supremum of Hausdorff dimensions of probability $f$-invariant positive entropy measures on $J(f)$ is equal to the smallest exponent $\delta$ for which a Sullivan's conformal measure exists (i.e., a probability measure with Jacobian $\left|f^{\prime}\right|^{\delta}$ ). This equality was almost proved in [DU], and the above-mentioned version of Corollary A was just the missing link.

We end this introduction with a sketch of the proof of Theorem $A$ in the case there exists only one critical $c$ in $J(f)$ because in this case the main idea is very transparent. We consider the Riemann sphere with the standard conformal metric; the distances and $\left|f^{\prime}(x)\right|$ are considered with respect to it; for any $x \in J, r>0, B(x, r)$ denotes the ball with the origin at $x$ and radius $r$ in this metric.

Step 1 . There exists a constant $C>0$ such that, for every $n \geq 0, \operatorname{dist}\left(f^{n}(c), c\right)$ $>\exp -n C$. Otherwise $f^{n}$ maps $B=B(c, 2 \exp -n C)$ into itself and so the family $\left.f^{k n}\right|_{B}, k=1, \ldots$, is normal. Hence, $c \notin J$, a contradiction.

Step 2. Case (1). Assume that for a constant $C>0, x \in J$, and every $n$ large enough we have $\operatorname{dist}\left(f^{n}(x), c\right)>\exp -n C$. Then

$$
\limsup _{n \rightarrow \infty} \frac{1}{n} \log \left|\left(f^{n}\right)^{\prime}(x)\right| \geq-C .
$$

Otherwise the diameters of $f^{n}(B(x, \varepsilon))$ for $\varepsilon$ small enough shrink at least like $\left|\left(f^{n}\right)^{\prime}(x)\right| \tau^{n}$ for $\tau$ arbitrarily close to 1 due to the fact that the contraction is stronger than approaching by $f^{n}(x)$ the critical point (this is a standard Pesin's theory consideration). Again $\left.f^{n}\right|_{B(x, \varepsilon)}$ would be a normal family. Thus if $\chi(x)$ exists, we get $\chi(x) \geq-C$.

Case (2). If there exist $n$ arbitrarily large with $\operatorname{dist}\left(f^{n}(x), c\right) \leq \frac{1}{2} \exp -n C$, then for $C$ as in Step 1, there is a univalent branch $f_{\nu}^{-n}$ on $B=B(c$, exp-nC) such that $f_{\nu}^{-n}(B) \ni x$ and with the use of Koebe's distortion lemma we obtain

$$
\left|\left(f^{n}\right)^{\prime}(x)\right| \geq \text { Const } \operatorname{diam}(B)=\text { Const } \exp -n C,
$$

since, roughly speaking, $f_{\nu}^{-n}(B)$ cannot be larger than $\overline{\mathbb{C}}$. Hence, we again obtain (2), and if $\chi(x)$ exists we get $\chi(x) \geq-C$. 
The idea is that the "bad" case where $f^{n}(x)$ is close to $c$ happens to be "good" because the critical point is the safest from being contaminated by critical values for iterates of $f$. The model situation is the subexpanding case where the critical values cannot approach $c$ at all.

Step 3. $\chi(x) \geq-C$ for a constant $C>0$ and $\mu$-almost every $x$ implies by the Birkhoff Ergodic Theorem the integrability of $\log \left|f^{\prime}\right|$ which implies $\chi \geq 0$. This is standard.

\section{Finiteness feAtures}

Here $f$ is a rational mapping of the Riemann sphere; we keep the notation from $\S 0$.

Lemma 1. Let $c \in$ Crit $\cap J$. There exists a constant $C_{1}>0$ such that, for every $\varepsilon, n>0$, if $f^{n}(B(c, \varepsilon)) \cap B(c, \varepsilon) \neq \varnothing$ then $n \geq C_{1} \log \frac{1}{\varepsilon}$.

Proof. We may assume $\varepsilon$ is small, if it is large $C_{1}$ takes care of it. Then $\operatorname{diam} f(B(c, 2 \varepsilon)) \leq 10 \varepsilon^{2}$ and $\operatorname{diam} f^{n}(B(c, 2 \varepsilon)) \leq 10 \varepsilon^{2} L^{n-1}$ where $L=$ $\sup _{x \in \overline{\mathbb{C}}}\left|f^{\prime}(x)\right|$. If $10 \varepsilon^{2} L^{n-1}<\varepsilon$, then as $f^{n}(B(c, 2 \varepsilon))$ intersects $B(c, \varepsilon)$ we obtain $f^{n}(B(c, 2 \varepsilon)) \subset B(c, 2 \varepsilon)$, so $c$ is in a domain of normality for the iterates $f^{n}$, which contradicts $c \in J$. Thus, $10 \varepsilon^{2} L^{n-1} \geq \varepsilon$; hence, $n \geq\left(\log \frac{1}{10 \varepsilon}\right) / \log L$, which proves our lemma.

Lemma 2 (finiteness lemma). There exists a constant $M>0$ such that for every $c \in \mathrm{Crit} \cap J$, for every $n \geq 0$ there are at most $M$ critical values for $f^{n}$ in $B=B(c, \exp -n)$.

Proof. Suppose that for $c_{1} \in$ Crit , $k>l \geq 0$, we have $f^{k}\left(c_{1}\right), f^{l}\left(c_{1}\right) \in B$. Then by Lemma $1 k-l \geq C_{1} \log \left(\frac{1}{\exp -n}\right)=C_{1} n$. So for $M=\left(C_{1}^{-1}+1\right) \sharp$ Crit Lemma 2 is satisfied.

In the sequel we shall refer to the following Mañé's lemma [M]:

Lemma 3 (Mañé's lemma). Given $\varepsilon>0,0<k<1, c>0$, and $N>0$ there exists $\delta>0$ such that, if for a disc $B=B(x, \delta)$ we have $\operatorname{dist}(B, p)>c$ for every neutral rational or attracting periodic point $p$ and for some $n>0$ and $a$ component $V$ of $f^{-n}(B)$ there are not more than $N$ critical points of $f^{n}$ in $V$, then diam $W \leq \varepsilon$ for every component $W$ of $f^{-n}(B(x, k \delta)) \cap V$.

In fact, the inductive consideration proving the next lemma also proves Mañé's lemma for $N>0$ if one has it already for $N=0$.

Lemma 4 (bounded distortion lemma). There exists $C_{2}>0$, such that for every $c \in \mathrm{Crit} \cap J$ and $n \geq 0$ there exists $r, \frac{1}{2}<r<1$, such that for every $j, 0 \leq$ $j \leq n$, every component $D_{r}$ of $f^{-n}\left(B_{r}\right)$, where we write $B_{\tau}=B(c, \tau \exp -n)$ for every $\tau, 0<\tau \leq 1$, the following holds:

for every two points $y \in \partial D_{r}, z \in D_{r} \cap f^{-n}\left(B_{1 / 2}\right)$

$$
\operatorname{dist}\left(f^{j}(y), f^{j}(z)\right) \geq C_{2} \operatorname{diam} f^{j}\left(D_{r}\right) .
$$

Proof. Let $D$ be an arbitrary component of $f^{-n}\left(B_{3 / 4}\right)$. We need to consider only large $n$. So due to Lemma 2 and Mañé's lemma we may assume that the diameters of all $f^{j}(D), j=0, \ldots, n$, are small. Due to Lemma 2 there exist $\frac{1}{2} \leq r_{1}<r_{2} \leq \frac{3}{4}, r_{2}-r_{1} \geq \frac{1}{4 M}$ such that there are no critical values for $f^{n}$ 
in $\operatorname{cl} B_{r_{2}} \backslash B_{r_{1}}$. Consider an arbitrary component $D_{r_{2}}$ of $f^{-n}\left(B_{r_{2}}\right)$ in $D$. For $r_{1} \leq \tau \leq r_{2}$ denote $f^{-n}\left(B_{\tau}\right) \cap D_{r_{2}}$ by $D_{\tau}$.

Observe that all sets $f^{j}\left(D_{r_{2}}\right)$ are simply connected, $f^{j}\left(D_{r_{2}} \backslash \operatorname{cl} D_{r_{1}}\right)$ are topological annuli, and the degree of $f^{n}$ on $D_{r_{2}} \backslash \operatorname{cl} D_{r_{1}}$ is at most $d^{M \sharp C r i t}$ where $d$ majorizes multiplicities of $f$ at critical points.

We prove it by induction. For $j=n$ everything has been assumed. Assume the above assertion for $j \leq n$. Then $f^{j-1}\left(D_{r_{2}}\right)$ contains at most one critical point for $f$ because it has small diameter. This critical point is in fact in $f^{j-1}\left(D_{r_{1}}\right)$. So $f^{j-1}\left(D_{r_{2}}\right)$ is simply connected, $f^{j-1}\left(D_{r_{2}} \backslash \mathrm{cl} D_{r_{1}}\right)$ is a topological annulus, and the degree of $f$ on it is the multiplicity of it at the critical point. Formally from Lemma 2 it follows that we meet critical point with $f^{j}\left(D_{r_{2}}\right)$ for at most $M \sharp$ Crit number of $j$ 's. (We multiply $M$ by $\sharp$ Crit as the orbit of one critical point may hit another critical point so they give the same critical value for $f^{n}$ in $B_{r_{2}}$. In fact, from the proof of Lemma 2 it follows that we can omit the factor $\sharp$ Crit as we shall in the sequel.)

Let

$$
r^{\prime \prime}=r_{2}-\frac{1}{3}\left(r_{2}-r_{1}\right), \quad r^{\prime}=r_{1}+\frac{1}{3}\left(r_{2}-r_{1}\right) .
$$

The distortion (i.e., supremum of the ratios of the absolute values of derivatives over all pairs of points) of each branch of $f^{-n(n-j)}$ on, say, each half of the annulus $B_{r^{\prime \prime}} \backslash B_{r^{\prime}}$ (for example, $0 \leq \operatorname{Arg}(z-c) \leq \pi$ or $\pi \leq \operatorname{Arg}(z-c) \leq 2 \pi$ ) to $f^{j}\left(D_{r^{\prime \prime}} \backslash D_{r^{\prime}}\right)$ is bounded by a constant depending only on $M$ (use Koebe's distortion lemma). Finally we have degree of $f^{n-j}$ on $f^{j}\left(D_{r^{\prime \prime}} \backslash D_{r^{\prime}}\right)$ bounded by $d^{M}$. This yields the lemma for $r=r^{\prime \prime}$.

Lemma 5. There exist $\kappa, C_{3}>0$ such that for every $n, x$ if $f^{n}(x) \in B_{1 / 2}$ then there exist $j, \Delta$ satisfying $0 \leq j<j+\Delta \leq n, \Delta \geq n \kappa$ such that

$$
\left|\left(f^{\Delta}\right)^{\prime}\left(f^{j}(x)\right)\right| \geq C_{3} \exp -2 n .
$$

Proof. Take $D_{1 / 2} \ni x$ as in Lemma 4 . We have

$$
\operatorname{diam} f^{n}\left(D_{1 / 2}\right) / \operatorname{diam} D_{1 / 2} \geq(\exp -n) /(2 \operatorname{diam} \overline{\mathbb{C}}) .
$$

Hence, for some $T \leq M+1$

$$
\prod_{t=1}^{T}\left(\frac{\operatorname{diam} f^{j_{t}}\left(D_{1 / 2}\right)}{\operatorname{diam} f^{j_{t-1}+1}\left(D_{1 / 2}\right)}\right) \geq \text { Const exp }-n
$$

where $j_{0}=0, j_{T}=n$, and, for $t=1, \ldots, T-1, j_{t}<j_{t+1}$ are consecutive integers for which $f^{j_{t}}\left(D_{r}\right)$ intersects Crit ( $r$ from Lemma 4).

Denote $\Delta_{t}=j_{t}-j_{t-1}-1$. By Lemma 4 and Koebe's distortion lemma $f^{\Delta_{t}}$ for every $t$ has distortion on $f^{j_{t-1}+1}\left(D_{1 / 2}\right)$ bounded by a constant depending only on $C_{2}$. Hence from (4) for a constant $C_{4}$ depending only on $C_{2}$ we get

$$
\prod_{t=1}^{T}\left|\left(f^{\Delta_{t}}\right)^{\prime}\left(f^{j_{t-1+1}}(x)\right)\right|>C_{4}^{T} \exp -n .
$$

If each $A_{t}=\left|\left(f^{\Delta_{t}}\right)^{\prime}\left(f^{j_{t-1}+1}(x)\right)\right|$ for $\Delta_{t}>\kappa n$ were smaller than exp-2n ( $\kappa$ will be specified below), then

$$
\prod A_{t} \leq \prod_{\Delta_{t}>\kappa n} A_{t} \prod_{\Delta_{t} \leq \kappa n} A_{t} \leq(\exp -2 n) L^{\kappa n T} .
$$


We used the fact that there exists $\Delta_{t}>\kappa n$, which holds if $\kappa n T+T<n$. If we assume additionally $1>\kappa T \log L$, then we get $\prod A_{t}<\exp -n(1+\tau)$ for some $\tau>0$, which contradicts (5) for $n$ large.

\section{2. $\chi(x) \geq 0$ FOR A RATIONAL MAPPING OF $\overline{\mathbb{C}}$}

In this section we shall prove Theorem $\mathrm{A}$. We start with a lemma standard in Pesin theory [P]:

Lemma 6. For every $x \in \overline{\mathbb{C}}$ if

$$
\limsup _{n \rightarrow \infty} \frac{1}{n}\left(\log \left|\left(f^{n}\right)^{\prime}(x)\right|-\log \operatorname{dist}\left(f^{n}(x), \text { Crit }\right)\right)<0
$$

then there exists $\tau>0$ such that

$$
\lim _{n \rightarrow \infty} \operatorname{diam} f^{n}(B(x, \tau))=0
$$

more precisely,

$$
\lim _{n \rightarrow \infty} \frac{1}{n} \log \frac{\operatorname{diam} f^{n}(B(x, \tau))}{\left|\left(f^{n}\right)^{\prime}(x)\right|}=0 .
$$

Proof. Denote $A=\limsup \sup _{n \rightarrow \infty} \log \left(\left|\left(f^{n}\right)^{\prime}(x)\right|-\log \operatorname{dist}\left(f^{n}(x)\right.\right.$, Crit)). By (6) for every $\lambda>1$ satisfying $\log \lambda<|A|$ and for every $\varepsilon>0$ there exists $\tau>0$ such that for every $n \geq 0$

$$
\frac{\tau\left|\left(f^{n}\right)^{\prime}(x)\right| \lambda^{n}}{\operatorname{dist}\left(f^{n}(x), \text { Crit }\right)}<\varepsilon .
$$

Assume that $\varepsilon<1$. We have for every $y, z \in B_{n}:=B\left(f^{n}(x), \tau\left|\left(f^{n}\right)^{\prime}(x)\right| \lambda^{n}\right)$ the inequality

$$
\left|\frac{\left|f^{\prime}(y)\right|}{\left|f^{\prime}(z)\right|}-1\right| \leq \frac{\sup _{a, b \in B_{n}}\left|f^{\prime}(a)\right|-\left|f^{\prime}(b)\right|}{\inf _{a \in B_{n}}\left|f^{\prime}(a)\right|}:=R_{n} .
$$

We have diam $B_{n}$ converging to 0 exponentially fast. So in the case $B_{n}$ is far from Crit, the ratio $R_{n}$ is exponentially small. In the opposite case choose a closest critical point to $B_{n}$. Denote the multiplicity of $f$ at this critical point by $d$. We have

$$
R_{n} \leq \text { Const } \frac{\operatorname{dist}\left(B_{n}, \text { Crit }\right)^{d-2} \operatorname{diam} B_{n}}{\operatorname{dist}\left(B_{n}, \text { Crit }\right)^{d-1}} \leq \text { Const } \varepsilon .
$$

For $\varepsilon$ small enough this implies $f\left(B_{n}\right) \subset B_{n+1}$, so $f^{n}(B(x, \tau)) \subset B_{n}$ and diameters converge to 0 , which proves (7).

Taking $\lambda$ arbitrarily close to 1 one obtains the inequality $\leq$ in (8). The other side of the inequality follows from the existence of a universal bound for the distortions of all $f^{n}$ on $B(x, \tau)$.

Corollary. If $x \in J$ and $\operatorname{dist}\left(f^{n}(x)\right.$, Crit $) \geq \exp -n \delta$ for all $n$ large enough then $\chi(x) \geq-\delta$.

Proof. If the assertion were false then by Lemma 6 we would find $\tau$ for which (7) holds. Hence, the family $f^{n}$ would be normal on $B(x, \tau)$, which is contrary to the assumption $x \in J$. 
Proof of Theorem A. First we prove that there exists a constant Const such that for $\mu$-almost every $x \in J$ we have $\chi(x) \geq$ Const $>-\infty$. Let $E$ be the set in $J$ on which $\chi$ exists (we allow the value $-\infty$ ). By the Birkhoff Ergodic Theorem $\mu(E)=1$. Fix an arbitrary $\vartheta>0$. For every $C>0$ let $E(C)$ denote the set of $x \in E$ such that for every $n \geq 0$

$$
\begin{aligned}
& \log \left|\left(f^{n}\right)^{\prime}(x)\right|<n(\chi(x)+\vartheta)+C \quad \text { if } \chi(x)>-\infty, \\
& \text { or } \\
& \log \left|\left(f^{n}\right)^{\prime}(x)\right|<-n \vartheta^{-1}+C \quad \text { if } \chi(x)=-\infty .
\end{aligned}
$$

Of course $\bigcup_{C>0} E(C)=E$. Fix an arbitrary $C$ with $\mu(E(C))>0$. Consider the function $\Phi$ equal to 1 on $E(C)$ and 0 outside. Again by the Birkhoff Ergodic Theorem for almost every $x \in E(C)$ there exists a nonzero limit $\lim _{n \rightarrow \infty} \frac{1}{n} \sum_{j=0}^{n-1} \Phi\left(f^{j}(x)\right)$; hence, for all $j$ large enough (depending on $x$ ) we have

$$
n_{j+1}(x)-n_{j}(x)<\frac{\kappa}{2} n_{j}
$$

where $n_{j}(x)$ denote all consecutive integers for which $f^{n_{j}(x)}(x) \in E(C)$ and $\kappa$ is from Lemma 5 . We now have two possibilities:

1. Suppose that for every $n$ large enough $\operatorname{dist}\left(f^{n}(x)\right.$, Crit $)>\frac{1}{2} \exp -n$. In this case $\chi(x) \geq-2$ by the Corollary.

2. Suppose now that there exist $n$ arbitrarily large and $c \in$ Crit $\cap J$ such that $f^{n}(x) \in B\left(c, \frac{1}{2} \exp -n\right)$. Then by Lemma $5\left(f^{\Delta}\right)^{\prime}\left(f^{j}(x)\right) \geq C_{3} \exp -2 n$. As $\Delta \geq \kappa n$ we find by (10) $t \geq 0, t<\frac{\kappa}{2} n$ such that $f^{j+t}(x) \in E(C)$. We have

$$
\left|\left(f^{\Delta-t}\right)^{\prime}\left(f^{j+t}(x)\right)\right| \cdot L^{t} \geq\left|\left(f^{\Delta}\right)^{\prime}\left(f^{j}(x)\right)\right|
$$

(recall that $\left.L=\sup \left|f^{\prime}\right|\right)$. Hence,

$$
\left|\left(f^{\Delta-t}\right)^{\prime}\left(f^{j+t}(x)\right)\right| \geq L^{-\kappa n / 2} C_{3} \exp -2 n
$$

and in view of (9)

$$
\exp \left((\Delta-t)\left(\chi\left(f^{j+t}(x)\right)+\vartheta\right)+C\right) \geq L^{-\kappa n / 2} C_{3} \exp -2 n
$$

or

$$
\exp \left(-(\Delta-t) \vartheta^{-1}+C\right) \geq L^{-\kappa n / 2} C_{3} \exp -2 n
$$

in the case $\chi\left(f^{j+t}(x)\right)=-\infty$

Hence, as $\chi$ is $f$-invariant and as we may restrict our consideration only to $\chi+\vartheta$ negative we get

$$
n \frac{\kappa}{2}(\chi(x)+\vartheta)+C \geq-2 n+\log C_{3}-n \frac{\kappa}{2} \log L
$$

or a corresponding inequality for $\chi=-\infty$.

Taking $\vartheta$ arbitrarily small (positive) and $n$ large we see that the case $\chi=$ $-\infty$ leads to a contradiction, so we arrive at

$$
\chi(x) \geq-4 / \kappa-\log L \text {. }
$$


In particular, we have proved that $\int \log \left|f^{\prime}(x)\right| d \mu(x)=\int \log \chi(x) d \mu(x)>$ $-\infty$; i.e., $\log \left|f^{\prime}\right|$ is $\mu$-integrable.

Now we shall prove that, for almost every $x, \chi(x) \geq 0$.

For an arbitrary $c \in \mathrm{Crit} \cap J$ and every $\delta>0$ denote $B_{n}=B(c, \exp -n \delta)$. Then for $n_{0}$ large enough, denoting the multiplicity of $f$ at $c$ by $d$, we have

$$
\begin{aligned}
-\infty & <\int_{B_{n_{0}}} \log \left|f^{\prime}\right| d \mu \leq \text { Const } \sum_{n \geq n_{0}} \int_{B_{n} \backslash B_{n+1}} \log \operatorname{dist}(x, c)^{d-1} d \mu(x) \\
& \leq \operatorname{Const}(d-1) \sum_{n \geq n_{0}}(-n \delta) \mu\left(B_{n} \backslash B_{n+1}\right) \\
& =-\operatorname{Const}(d-1) \delta \sum_{n \geq n_{0}} \mu\left(B_{n}\right) .
\end{aligned}
$$

So the series $\sum \mu\left(B_{n}\right)$ is convergent and, by the $f$-invariance of $\mu$, $\sum \mu\left(f^{-n}\left(B_{n}\right)\right)$ is convergent. So by the Borel-Cantelli lemma, for almost every $x, f^{n}(x) \notin B(c, \exp -n \delta)$ for $n$ sufficiently large. Doing the same for every $c \in$ Crit $\cap J$ we obtain $\operatorname{dist}\left(f^{n}(x)\right.$, Crit $) \geq \exp -n \delta$ for every $\delta>0$ and $n$ sufficiently large. So by the Corollary we obtain $\chi(x) \geq 0$.

Proof of Corollary A. Suppose the assertion of Corollary A is false. Then there exist $E \subset J$, an integer $n_{0}$, and $0<C<1$ such that $\mu(E)>0$ (in fact, $\mu(E)$ arbitrarily close to 1$)$, and for every $x \in E, n \geq n_{0}$ one has $\left|\left(f^{n}\right)^{\prime}(x)\right|<C<1$.

Given $x$ denote $J(n)=\left\{j: 0 \leq j \leq n, f^{j}(x) \in E\right\}$. By the Birkhoff Ergodic Theorem for almost every $x \in E$ there exists $\alpha=a(x)>0$ such that $\#(J(n)) \geq \alpha n$ for all $n$ large enough. So taking into account only each $n_{0}$ th $j$ from $J(n)$ and next neglecting the last one and indexing them $j_{1}, j_{2}, \ldots, j_{m}$ we obtain under the convention $j_{0}=0, j_{m+1}=n$

$$
\left|\left(f^{n}\right)^{\prime}(x)\right|=\prod_{s=0}^{m} \mid\left(f^{j_{s+1}-j_{s}}\left(f^{j_{s}}(x)\right)\right)^{\prime}<C^{\alpha n / n_{0}-1} .
$$

This implies $\chi(x) \leq \frac{\alpha}{n_{0}} \log C<0$, which contradicts Theorem A.

\section{THE INTERVAL CASE}

The proof of Theorem B is basically the same as in the complex case, so we list only the places where there are differences.

1. The proof of the analogon of Lemma 1 (frequency lemma) should be changed because it is not sufficient to arrive at the situation $f^{n}(B(c, \varepsilon)) \subset$ $B(c, \varepsilon)$ contradictory in the complex case. In the interval case this happens even for $\varepsilon$ arbitrarily small, for unimodal $\infty$-renormalizable maps [CE]. The modified proof is as follows:

Fix $n>0$ and suppose that there exists a critical point $c$ not in a basin of attraction to a periodic sin:- or a neutral point such that

$$
f^{n}([c-\varepsilon, c+\varepsilon]) \cap[c-\varepsilon, c+\varepsilon] \neq \varnothing .
$$

Fix an arbitary $\varepsilon_{0}$ for which there exists a critical point $c_{0}$ so that (11) holds and

$$
\varepsilon_{0} \leq 2 \inf \{\varepsilon:(11) \text { holds for } \varepsilon \text { and some } c\}
$$


If $n<\log \frac{1}{10 \varepsilon_{0}} / \log L$ then for every $j=1, \ldots, n$ under the notation $J_{j}:=$ $f^{j}\left[\left(c_{0}-2 \varepsilon_{0}, c_{0}+2 \varepsilon_{0}\right]\right)$ we have

$$
\left|J_{j}\right| \leq 5 \varepsilon_{0}^{2} L^{n}<\varepsilon_{0} / 2 .
$$

So there is no critical point in $J_{j}$ for every $j=1, \ldots, n$. Otherwise, as in (11) $f^{n}\left(J_{j}\right) \cap J_{j} \neq \varnothing$, so the assumption (12) would not be satisfied.

We conclude that $f^{n}\left(J_{0}\right)$ lands in $J_{0}$ entirely right or left of $c_{0}$ and $f^{n}$ on $\left[c_{0}-2 \varepsilon_{0}, c\right]$ and on $\left[c, c_{0}+2 \varepsilon_{0}\right]$ is monotone. This implies the existence of a sink of period $n$ or $2 n$ in $J_{0}$ which attracts $c_{0}$, a contradiction. We conclude that $n \geq \log \frac{1}{20 \varepsilon} / \log L$ if (11) is satisfied.

2. The analogon of Lemma 3 (Mañé's lemma) holds even with $k=1$. However, in the presence of infinitely many sinks or neutral points one needs to fix a closed set $A \subset J^{\mathbb{R}}$ not containing sinks and neutral points and instead of assuming distance of $B(x, d)$ from them greater than $c$ one assumes $\operatorname{dist}(B(x, d), A)<d$.

We prove this for $N=0$ (then the case $N>0$ is very easy). If a sequence of intervals $\left(I_{n}\right)$ satisfies the properties $\operatorname{diam} I_{n} \geq$ Const $>0, \operatorname{diam} f^{n}\left(I_{n}\right) \rightarrow 0$, and $\operatorname{dist}\left(f^{n}\left(I_{n}\right), A\right) \rightarrow 0$, then all the intervals from a subsequence $I_{n_{j}}$ of $\left(I_{n}\right)$ contain a nondegenerate interval $J$ which is a homterval; i.e., $f^{n}(J)$ do not contain critical points for all $n=0,1, \ldots$ and $\operatorname{diam} f^{n_{j}}(J) \rightarrow 0$. So by [MMS] or [BL] $f^{n}(J)$ converge to a sink or a neutral point, contrary to the convergence of a subsequence to $A$.

3. In the proofs of the analogons of Lemma 4 and Lemma 5 we use Koebe's distortion lemma in the interval version [S, MS]. Denote the interval being the component of $f^{-(n-j)}\left(B_{r_{2}}\right)$ containing $f^{j}\left(D_{r_{2}}\right)$ by $D^{j}, J=0, \ldots, n,\left(r_{2}\right.$ from the proof of Lemma 4) and the analogous sets for $r_{1}$ by $D^{\prime j}$. We apply Koebe's distortion lemma to each $f^{-\Delta_{t}}: D^{j_{t+1}} \rightarrow D^{j_{t}+1}$ (notation $\Delta_{t}$ from the proof of Lemma 5). This is allowed due to the fact that the multiplicity of the covering by the family of $\left(D^{j}\right), j=j_{t}+1, \ldots, j_{t+1}$, is bounded by a universal constant (finiteness lemma). $D^{j_{t}}$

Finally for each $t$ we have for each component $A$ of $f^{-1}\left(D^{j_{t}+1} \backslash D^{j_{t}+1}\right)$ in

$$
\frac{\operatorname{diam} A}{\operatorname{diam} D^{j_{t}}} \geq \text { Const } \frac{\operatorname{diam} f(A)}{\operatorname{diam} D^{j_{t}+1}},
$$

where Const depends only on the nonflatness at the critical point involved (see Figure 1).

4. In the proof of the analogon of the Corollary from $\S 2$ the argument of normality should be replaced by the existence of a homterval $J$ of $\operatorname{diam} f^{n}(J) \rightarrow 0$ so by [BL] $f^{n}(J)$ converges to a sink or a neutral point (as at point 2).

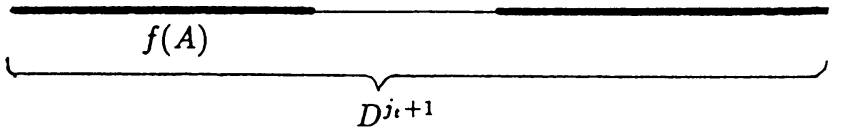

FIGURE 1 


\section{ADDED IN PROOF}

Theorem B follows easily from the "specification property", more precisely from Corollary 5 of A. M. Blokh, Decomposition of dynamical systems on an interval, Russian Math. Surveys 38 (1983), no. 5, 133-134. I owe this observation to F. Hofbauer.

\section{REFERENCES}

[BL] A. M. Blokh and M. Yu. Lyubich, Non-existence of wandering intervals and structure of topological attractors of one-dimensional dynamical systems II. The smooth case, Ergodic Theory Dynamical Systems 9 (1988), 751-758.

[CE] P. Collet and J.-P. Eckmann, Iterated maps on the interval as dynamical systems, Birkhäuser, Basel, Boston, and Stuttgart, 1980.

[DU] M. Denker and M. Urbański, On Sullivan's conformal measures for rational maps of the Riemann sphere, Nonlinearity 4 (1991), 365-384.

[GPS] P. Grzegorczyk, F. Przytycki, and W. Szlenk, On iterations of Misiurewicz's rational maps on the Riemann sphere, Ann. Inst. H. Poincaré Phys. Théor. 53 (1990), 431-444.

[H] M. Herman, Exemples de fractions rationnelles ayant une orbit dense sur la sphère de Riemann, Bull. Soc. Math. France 112 (1984), 93-142.

[M] R. Mañé, On a theorem of Fatou, preprint, 1991.

[MMS] M. Martens, W. de Melo, and S. van Strien, Julia-Fatou-Sullivan theory for real one dimensional dynamics, preprint, Delft, 1988.

[MS] W. de Melo and S. van Strien, A structure theorem in one-dimensional dynamics, Ann. of Math. (2) 129 (1989), 519-546.

[P] Ja. Pesin, Characteristic Lyapunov exponents and smooth ergodic theory, Russian Math. Surveys 32 (1977), 55-114.

[S] S. van Strien, Hyperbolic and invariant measures for general $C^{2}$ interval maps satisfying the Misiurewicz condition, preprint, Delft, 1987.

Institute of Mathematics, Polish Academy of Sciences, Ul. Śniadeckich 8, 00950 Warszawa, Poland

E-mail address: Feliksp@impan.impan.gov.pl 